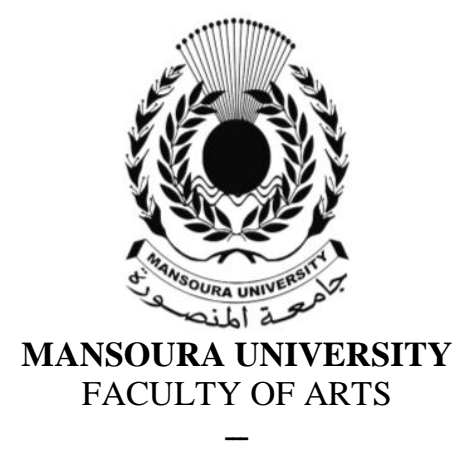

\title{
L'ÉTUDE DE PAUSES DANS LE DISCOURS POLITIQUE KURDE, LE CAS DU PREMIER MINISTRE NECHIRVAN BARZANI
}

\author{
By \\ Dr. Ibrahim Khaleel ABDULBAQI \\ Lecteur au département de français - Faculté des langues \\ Université de Salahaddine
}

Journal Of The Faculty Of Arts- Mansoura University

$53^{\text {rd }}$ ISSUE- Aug. 2013 


\title{
L'ÉTUDE DE PAUSES DANS LE DISCOURS POLITIQUE KURDE, LE CAS DU PREMIER MINISTRE NECHIRVAN BARZANI
}

\author{
Dr. Ibrahim Khaleel Abdulbaqi \\ Lecteur au département de français - Faculté des langues \\ Université de Salahaddine
}

\section{Introduction}

Être un bon orateur politique n'exige pas seulement la maîtrise des outils segmentaux de la parole mais demande également la maîtrise des outils suprasegmentaux de la langue. Les hommes politiques se rendent compte de l'importance de ces outils prosodiques quand il s'agit de persuader et de convaincre lepublic. Comme c'est le cas chez tous les hommes politiques, les hommes politiques kurdes essaient, de leur côté, de profiter de ces éléments prosodiques pour des raisons argumentatives et discursives. Parmi les outils prosodiques suprasegmentaux, nous allons jeter la lumière sur les types et le rôle de la pause dans le discours politique oral kurde.

Ainsi, l'objectif de cette recherche est de franchir un premier pas pour définir la pause dans le discours politique kurde à l'oral. Avant d'entamer l'analyse des différents types de pause abordés dans cette recherche, il parait intéressant d'en donner quelques définitions. La pause est définie comme "une cessation de l'activité verbale qui se traduit au niveau acoustique par une interruption du signal sonore » (D. Duez, 1999 : 91). Pour sa part, A. Dister la définit comme "une interruption perçue de continuum du signal sonore dans une prise de parole » (Dister, $2008: 166$ ). Quant à A. Lacheret-Dujour \&F. Beaugendre, ils écrivent que les pauses sont «des mécanismes biologiques liés à des prises de souffle nécessaires pendant la production de la parole» (Lacheret-Dujour \&Beaugendre, 1999 :48). De leur côté, M-A. Morel \&L. Danon-Boileau décrivent le terme pause-silence de la manière suivante : «la pause silencieuse permet, sur la base d'une attention supposée partagée, d'homogénéiser ce qui précède et de thématiser ce qui va suivre » (Morel \&Danon-Boileau, $1998: 10$ ). La pause est donc un élément prosodique non sonore qui interrompt momentanément les constituants sonores du discours afin de leur conférer une sorte d'homogénéisation sonore. En fait, la pause permet au locuteur d'organiserl'articulation de ses énoncés. Elle lui offre le temps nécessaire pour produire l'énoncé qui suit.Duez (1999 : 97) observe que les pauses sont 
utilisées par les hommes politiques pour exercer un effet expressif sur l'auditeur.Ces hommes politiques se servent de la durée de la pause pour accélérer ou ralentir le débit de leur élocution afin de réaliser certaines stratégies argumentatives et persuasives.

\section{Quelques éléments de phonétique kurde}

La langue kurde est une langue indoeuropéenne, elle possède deux principaux dialectes (Kermanji et Sorani). C'est la langue du peuple kurde qui vit : en Irak, en Iran, en Turquie et en Syrie. Comme toutes les autres langues, la langue kurde possède un système phonétique comportant des consonnes et des voyelles. Il ya donc huit voyelles et vingt-quatre consonnes selon certains linguistes kurdes et vingt-huit selond'autres. Les consonnes sont divisées, comme les consonnes françaises, en consonnes voisées [b,d,g] et non-voisées [p,t,k] ( Gazi F. Wais, 1984 :56). Quant à la prosodie kurde, elle possède les mêmes éléments suprasegmentaux que la langue française (intonation, accent, rythme et pause). En ce qui concerne la pause en kurde, nous observons trois types de pausesmais qui sont appelées différemment :

- Westan : une pause longue qui indique la fin de phrase.

- Halwsan: une pause courte qui marque la fin d'uneproposition ou d'un groupe de mots à l'intérieur de la phrase.

- Pechou: une pause d'aspiration qui permet au locuteur de reprendre la parole (AbdulwahabKh Moussa, 2009 :36-38).

En réalité, nous n'avons pas trouvé des références linguistiques kurdes qui abordent la pause de focalisation alors qu'elle est souvent utilisée dans le discours politique kurde.C'est un champ nouveau du domaine de recherche prosodique en langue kurde qui s'ouvre alors.

\section{Les types de pauses rencontrées lors de la recherche}

Dans notre corpus, nous allons aborder quatre types de pauses: pause courte,pause longue, pause d'hésitation et pause de focalisation. Les pauses courtes sont celles que le locuteur fait entre les groupes de mots. Les pauses longues, elles-mêmes, possèdent un rôle syntaxique lorsqu'elles indiquent la fin de la phrase kurde. Les pauses d'hésitation se trouvent à l'intérieur de la phrase et sont marquées par une durée plus longue que les pauses courtes. Elles sont réalisées lorsque le locuteur produit un énoncé incomplet et interrompu suivi d'une autocorrection ou d'un faux départ (Duez, 1999 :93). Les pauses de focalisation sont des pauses manifestement 
longues que le locuteur place devant un mot qui lui semble important (Ibid.92).

\section{Le développementde la recherche}

Dans cette recherche, nous avons choisi comme corpus un discours politique du Premier Ministre kurde M. Nechirvan Barzani. Ce discours concerne une conférence de presse à propos des manifestations qui se sont déroulées àSulaymani le 17 août 2011. Lors de cette conférence, M. Barzani analyseles événements et les conséquences qui ont suivi ces manifestations.

Le corpus consiste des extraits qui sont enregistrés d'abord en extension vidéo .flv convertis en extension .mp3 puis en.wav. Un découpage des séquences audio est fait afin d'illustrer les énoncés comportant des pauses. Pour ce faire, nous avons utilisé le logiciel Praat de Paul Boersma et David Weenink qui nous a permis de traiter facilement les données acoustiques des pauses.L'identification des pauses est faite par un procédé d'écoute et de réécoute du discours. Ensuite une segmentation des séquences porteuses de pauses, une description des énoncés transcrits ainsi qu'une transcription de la durée des pauses sont obtenues à l'aide dePraat.

Etant donné que le discours dure plus de vingt-trois minutes, nous n'avons transcrit que les séquences déjà segmentées. Les énoncés kurdes des séquences choisies sont transcris en lettres latines et sont accompagnés d'une traduction française proposée par l'auteur. Par ailleurs, toutes les pauses ne seront pas annotées sous Praat mais seulement quelques -unes pour illustration. Par contre, nous allons comptabiliser dans un tableau descriptif tous les types de pauses utilisées par le locuteur.

\section{Analyse des données prosodiques.}

Au travers de l'étude du discours de M.Nechirvan Barzani, nous avons remarqué qu'il utilise un langage soutenu enrichi par différents outils rhétoriques, argumentatifs et prosodiques. Ces derniers outils se complètent l'un à l'autre.Ce sont des équipements langagiers indispensables pour les hommes politiques. Lorsque le discours est oral et spontané, on s'aperçoit que l'homme politiqueattribue une grande importance à ce que l'énonciation aboutisseà bien transmettre le message sonore voulu. Et c'est là qu'on remarque le rôle crucial de la prosodie dans la transmission du message politique. Quant aux pauses utilisées par M. NechirvanBarzani, nous avons noté les quatre types de pauses mentionnés ci-dessus. Nous les aborderons de façon successive : 


\section{a. Les pauses courtes (P.C)}

Ce type de pause est très fréquent dans notre corpus et sert à poser les frontières entre les groupes syntaxiques d'une même phrase. Il permet également au locuteur de préparer le prochain énoncé. Examinons l'exemple suivant :

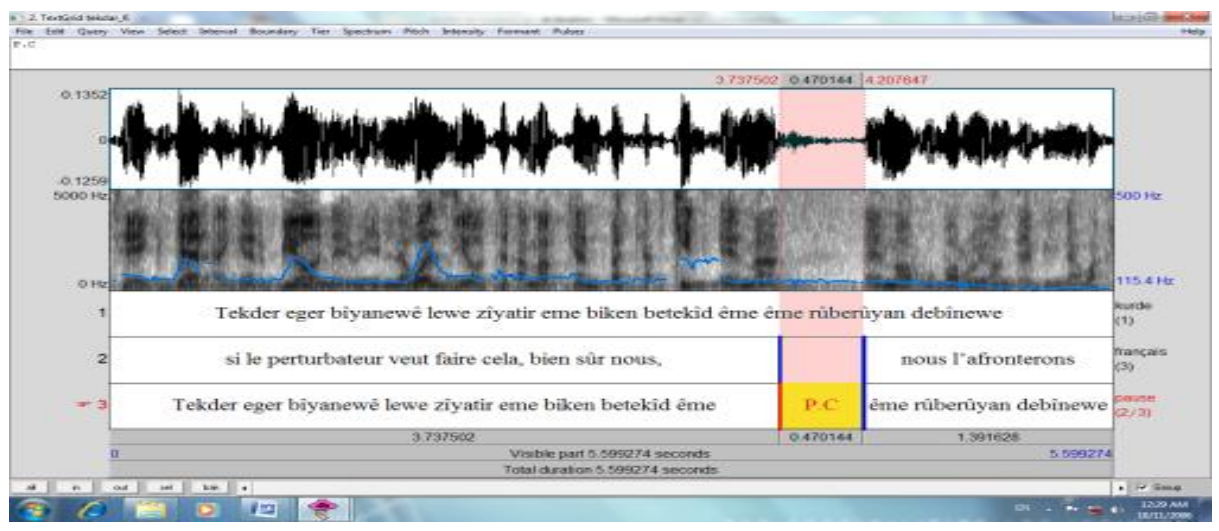

\section{Extrait 1 : pause courte}

Dans cet énoncé annoté, nous remarquons que la phrase conditionnelle« Tekderegerbîyanewêlewezîyatiremebikenbetekîdêmeêmerû berûyandebînewe » est constituée de deux propositions (principale et subordonnée) et que la pause courte est placée dans la proposition subordonnée. Elle sépareles deux groupes syntagmatiques et dure 470 millisecondes (ms). En même temps, cette pause sert à la répétition du pronom personnel pluriel «ême ». Le locuteur l'utilise donc entre les deux pronoms personnels afin de faciliter leur prononciation successive. La pause courte se rencontre régulièrement dans le discours de $\mathrm{M}$. Nechirvan Barzani à cause de son rôle syntaxique en tant que séparateur de groupes de mots. Sa durée est limitée entre 257 et $480 \mathrm{~ms}$ et est marquée par une courbe mélodique plate. Il est clair que le rôle de la pause courte se limite à - la séparation syntaxique des mots ou groupes de mots - dans la phrase kurde.

\section{b. Les pauses longues (P. L)}

Le second type de pause rencontré dans le discours de M. Nechirvan Barzani est la pause longue nommée en kurde «westan ». Cette pause est connue pour sa durée plus longue que celle de la pause courte et est placée à la fin de la phrase kurde. Voici un exemple qui illustre ce type de pause longue : 


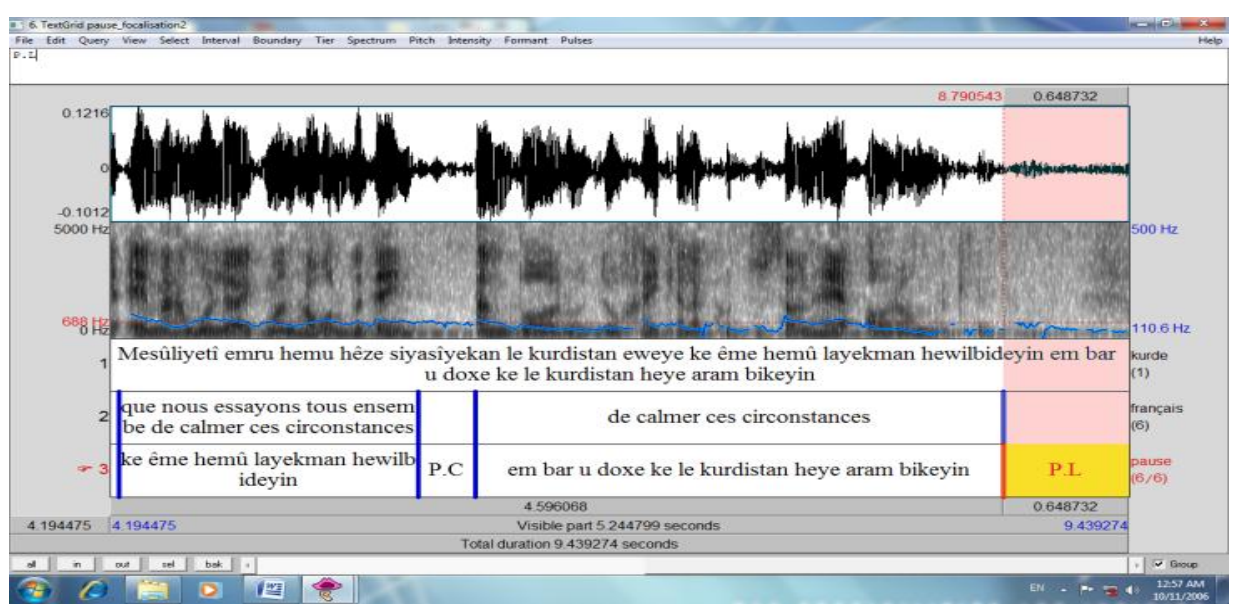

Extrait 2 : pause longue

Ici, nous remarquons que la phrase complexe « Mesûliyetîemruhemuhêzesiyasîyekanle kurdistaneweyekeêmehemûlayekmanhewilbideyinem bar $\mathrm{u}$ doxeke le kurdistanheyearambikeyin » comprend deux pauses : une pause courte et une pause longue. La pause longue est placée à la fin de la phrase. Elle dure $648 \mathrm{~ms}$,ce qui est sensiblement plus long que la pause courte. Dans ce corpus, la durée despauses longues que nous avons relevées se situe entre 491 et $650 \mathrm{~ms}$. Comme la pause courte, la pause longue a un rôle syntaxique dans la mesure où elle indique les limites entre les phrases kurdes. Les pauses longues sont plus fréquentes que les pauses courtes. Les premières sont toujours utilisées par le locuteur pour déterminer les frontières interphrastiques alors que les dernières sont dues au débit de parole. Autrement dit, les pauses courtes sont manifestes lorsque le locuteur s'exprime avec un débit ralenti alors qu'elles s'effacent lorsqu'il adopte un débit accéléré. Comme la pause courte, la pause longue est caractérisée par une courbe mélodique plate suivante une intonation montante ou descendante. En général, les pauses longues en kurde permettent à l'homme politique de structurer les phrases dans son discours oral.

\section{c. Les pauses d'hésitation (P.H)}

Les pauses d'hésitation sont « des pauses remplies par un silence et la suite sonore "euh», soit des syllabes allongées» (Lacheret-Dujour \&Beaugendre, 1999: 50). Cette pause, accompagnée par la marque d'hésitation « euh », est utilisée par le locuteur pour reformuler une phrase incertaine, mal prononcéeou un faux départ dans le processus de l'énonciation. Elle donne au locuteur le temps suffisant pour rectifier et pour 
reformuler son mot ou l'expression incorrecte. Pour mieux illustrer la pause d'hésitation, prenons l'exemple suivant :

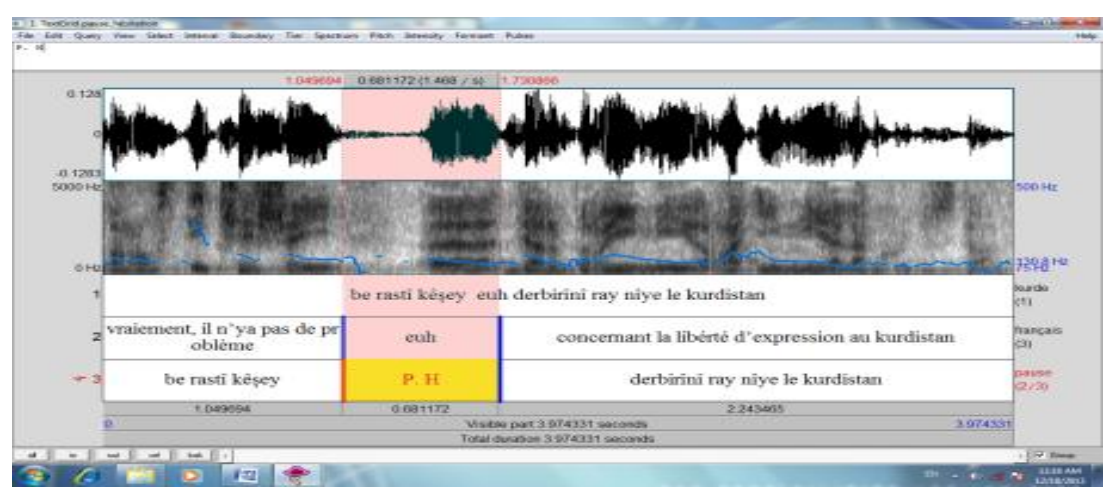

Extrait 3 : pause d'hésitation

Dans l'extrait 3, la pause d'hésitation est précédée par une marque d'hésitation «euh». Elle isole le mot «kêșey» (problème) des mots « derbirînî ray»(liberté d'expression).En examinant le contexte de la phrase, on remarque que la pause traduit l'hésitation du locuteur alorsqu'il est en train de choisirune des expressionsqui exprime l'idée de liberté de la pensée.Dans ce cas, si on compare la durée de la pause d'hésitation (681 $\mathrm{ms}$ ) avec la durée totale de l'énoncé (3974 ms), on se rend compte qu'elle est très longue et quele débit est manifestement ralenti.De même, elle se trouve à l'intérieur du premier ou second groupe syntaxique et apparaît dans le discours spontané lors d'une hésitation ou d'une auto-correction faite par le locuteur. Quant au mouvement mélodique qui accompagne la pause d'hésitation, on observe une pente mélodique descendante au début de la pause et une deuxième montante - descendante sur la marque d'hésitation « euh ».

\section{d. Les pauses de focalisation (P.F.)}

En fait, nous pouvons dire que les pauses de focalisation sont des pauses intentionnellement émises par le locuteur au moment où on ne les attend pas (P. Léon, 1992 : 103). Elles sont souvent utilisées devant un mot ou une expression que le locuteur veut mettre en relief. Elles sont également employées pour ralentir ou accélérer le débit de parole. Ce type de pause joue donc un rôle expressif lorsqu'elle permet au locuteur d'attirer l'attention de son public tout en focalisantsur un mot ou une expression importante. Cette particularité expressive rend le discours politique assez riche au niveau des pauses de focalisation.Par conséquent, le discours politique kurde est riche de ce type de pause et nous en avons relevé 
plusieurs durant notre analyse du discours politique de M. N. Barzani. Voici un exemple :

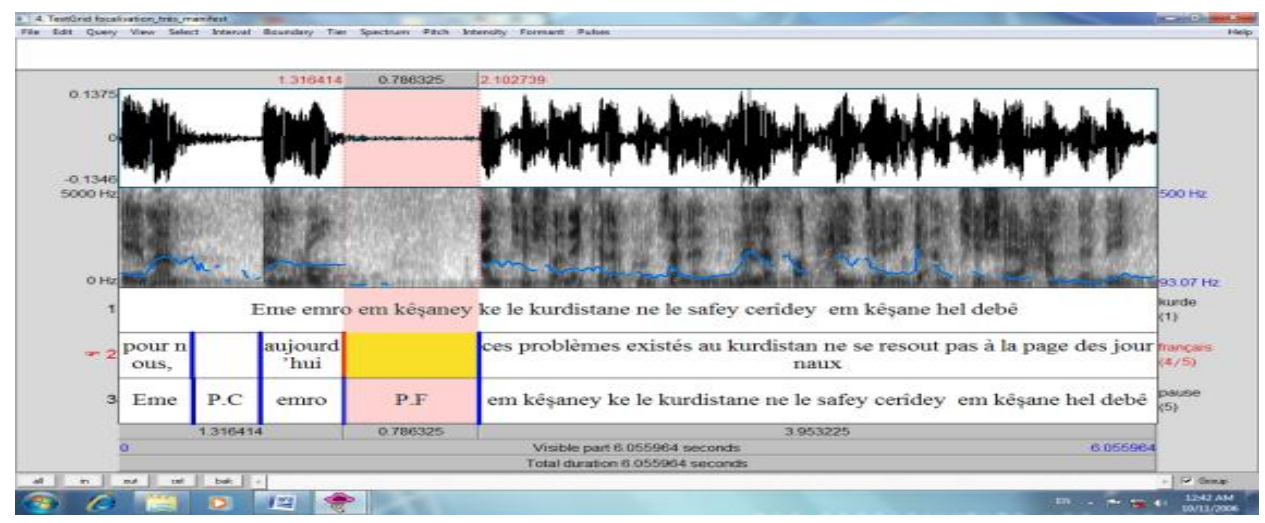

Extrait 4 : pause de focalisation (1/3)

Dans cet exemple, nous observons que le locuteur produit deux pauses dans son énoncé. La première est une pause courte $(393 \mathrm{~ms})$ et est placée après le pronom «ême»(nous). La seconde se trouve après le mot «emro» (aujourd'hui) et avant le mot «emkêșaney»(ces problèmes).En observant le mouvement mélodique de l'énoncé, nous relevonsune montée de courbe mélodique avant et après la pause de focalisation.

$\mathrm{Vu}$ sa longueur (786 ms) et sa position au début de l'énoncé et après la pause courte, nous pouvons dire que c'est une pause de focalisation utilisée pour des raisons discursives. Elle est employée par M. N. Barzanipour attirer l'attention des auditeurs sur l'importance du mot « kêșaney» (problèmes). De plus, sa place inattendue après une pause courte crée un suspens chez le public car il ne s'attend pas à une deuxième pause aussi rapidement. D'une part, cette technique le rend attentif à ce qui va être prononcé après cette pause. D'autre part, la pause permet au locuteur defocalisersur le mot «kêşaney» (problèmes)qui suit. Elle souligne donc une valeur sémantique lorsqu'elle assure la bonne transmission du message discursif.

Pour mieux illustrer le rôle de pause de focalisation dans le discours de M. Nechirvan Barzani,prenons un autre exemple : 


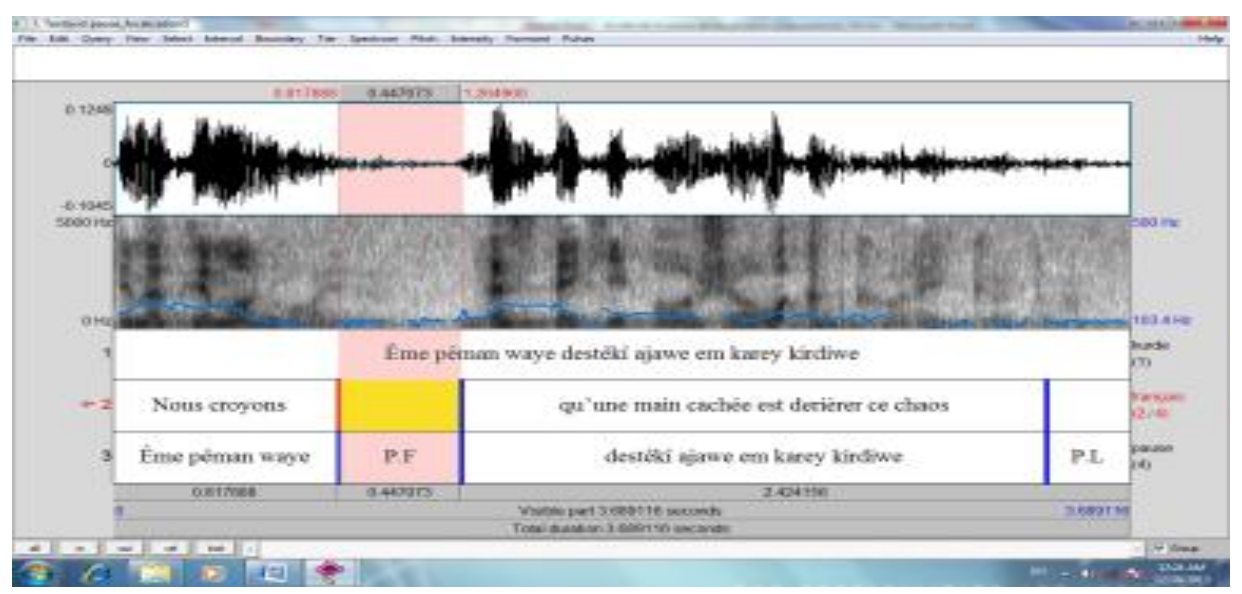

Extrait 5 : pause de focalisation (2/3)

Dans l'extrait ci-dessus, la pause de focalisation permet la séparation entre la proposition «Êmepêmanwaye»(nous croyons) et la proposition « destêkîajaweemkareykirdiwe» (qu'une main cachée est derrière ce chaos). En même temps, elle intervient à la place prévue d'une pause courte afin de réaliser un effet focalisateur. Le locuteur aurait pu mettre une pause courte pour séparer les deux groupes syntaxiques de la phrase, mais nous remarquons qu'il remplace la pause courte par une pause de focalisation assez longue pour mettre l'accent sur «destêkîajawe»(une main cachée). Nous pouvons défendrecette idée de remplacement parce que, d'un côté, la pause courte, en kurde, est toujours utilisée pour limiter les frontières syntaxiques à l'intérieur de la phrase et de l'autre, la durée maximale de la pause courte dans notre corpus est inférieure à $480 \mathrm{~ms}$, ce qui n'est pas le cas dans l'exemple ci-dessus. De plus, si nous comparons la longueur de l'énoncé $(2,937 \mathrm{~ms})$ à la durée de la pause de focalisation ( $447 \mathrm{~ms})$ et à celle de la pause longue $(303 \mathrm{~ms})$, nous trouvons que la pause de focalisation est remarquablement longue pour une phrase manifestement courte et qui aurait puse contenter d'une simple pause courte.

Dans les deux derniers exemples, nous avons vu que la pause de focalisation est employée pour attirer l'attention sur le mot ou l'expression qui précède. Elle sert également à focaliser sur ce qui est dit avant, comme le montre l'exemple suivant : 


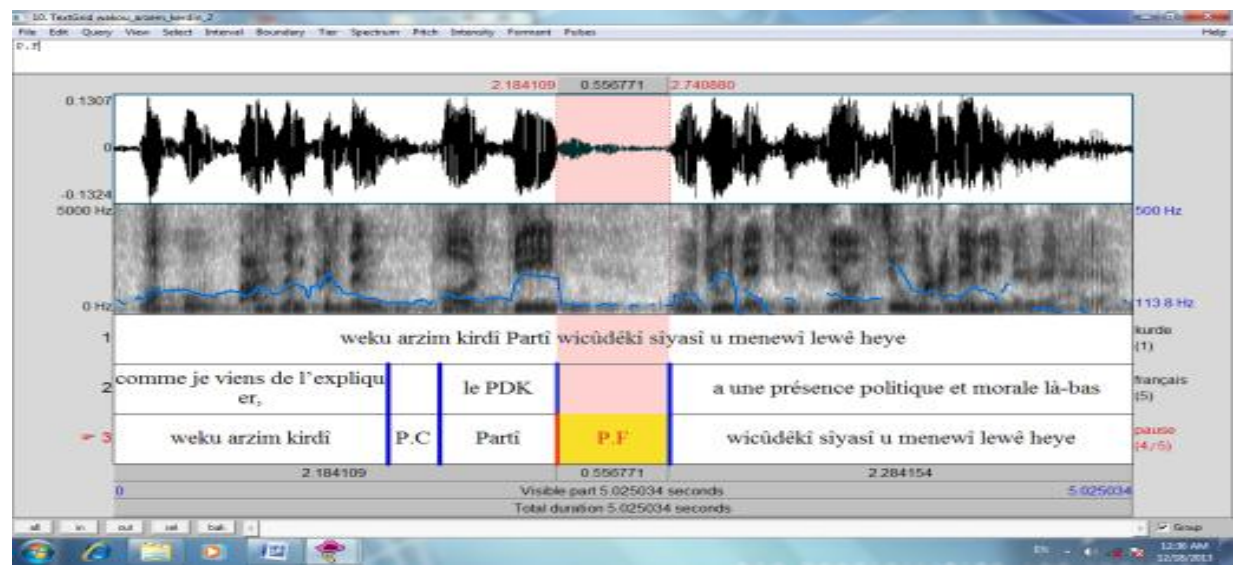

Extrait 6 : pause de focalisation (3/3)

Ici, la phrase étendue est constituée de deux propositions juxtaposées : « wekuarzemkirdî» (comme je viens de l'expliquer) et «Partîwicûdêkîsîyasî u menewîlewêheye»(le PDK a une présence politique et morale là-bas). La première proposition est marquée par une pause courte de $257 \mathrm{~ms}$. La seconde est caractérisée par une pause de focalisation située après le mot «Partî = le PDK ».D'après ce que nous avons vu dans les exemples précédents, cette pause aurait dû être placée avant le mot focalisé alors qu'ici le locuteur la place après. À ce propos, nous pouvons dire que la raison pour laquelle le locuteur effectue ce changement de place revient à la position du mot focalisé. Si nous regardons les extraits 4 et 5 , nous remarquerons que la pause de focalisation est placée avant le mot que le locuteur veut mettre en valeur. $\mathrm{Vu}$ le contexte de la phrase dans cet exemple, nous trouvons que le mot focalisé est le sigle «PDK» qui se trouve après la pause courte et avant la pause de focalisation.

De plus, dans les exemples précédents, et presque dans tous les cas de pause de focalisation, le mot que l'homme politiquemet en exergue se trouve à l'intérieur de la phrase alors que dans cet exemple, il se situe à l'initiale de la phrase. Plusieurs raisons nous amènent à cette conclusion : premièrement, le contexte de la phrase qui met l'accent sur le sens du sigle « PDK» qui signifie (le Parti Démocratique du Kurdistan) un des deux partis dirigeants au Kurdistan. C'est aussile parti dont M. Nechirvan Barzani est un des responsables politiques. Deuxièmement, l'élévation de la pente mélodique sur le mot situé entre les deux pauses par rapport à une baisse de la courbe sur les pauses elles-mêmes. Et finalement, les durées : le locuteur s'attarde pendant574 ms sur le mot «Partî» avant de faire une pause de 
focalisation de $556 \mathrm{~ms}$, d'une durée donc à peine inférieure. Là encore, l'homme politique se sert de cette pause de focalisation pour soigneusement présenter son mot-clef dans le but d'influencer son public.

Voici un tableau descriptif des types et du nombre de pauses localisées dans le discours de M. Barzani :

\begin{tabular}{|l|l|l|}
\hline Types de pause & Nombre & Durée moyenne \\
\hline Pause courte & 320 & $340 \mathrm{~ms}$ \\
\hline Pause longue & 97 & $570 \mathrm{~ms}$ \\
\hline Pause d'hésitation & 44 & $600 \mathrm{~ms}$ \\
\hline Pause de focalisation & 122 & $660 \mathrm{~ms}$ \\
\hline
\end{tabular}

\section{Conclusion}

En conclusion, nous avons vu dans cette recherche que la langue kurde, comme c'est le cas de la langue française et d'autres plusieurs langues, possède quatre types de pauses : la pause courte, la pause longue, la pause d'hésitation et la pause de focalisation.

Sur le plan syntaxique, la première est utilisée dans la langue kurde pour marquer les frontières syntaxiques entre les groupes de mots. La seconde détermine la fin de la phrase kurde. Par contre, la troisième a pour but de reformuler les phrases ou les énonciations difficiles du locuteur. Elle permet de reprendre le fil de sondiscours et de son argumentaire. La dernière assume un rôle essentiellement pragmatique.

Sur le plan prosodique, nous avons pu constater que la pause courte est marquée par une courbe mélodique plate et une durée moyenne de 340 ms. Comme la pause courte, la pause longue se caractérise également parune pente mélodiqueplate à la fin de la phrase mais elle se distingue par une durée moyenne de $570 \mathrm{~ms}$ qui est, de façon évidente, plus longue que celle de la pause courte. La pause d'hésitationse différencie par une montée de pente mélodique qui s'accompagne d'une reprise de souffle audible. La dernière se distingue des autres pauses par une montée saillante de courbe mélodique avant et après la pause.Cette montée baisse avant la pause de focalisation puis remonte après le mot focalisé.

Sur le plan pragmatique, nous avons pu découvrir qu'à part leur rôle syntaxique dans la structuration des phrases, la pause courte et la pause longue n'interviennent pas directement dans la stratégie discursive du locuteur politique kurde. Quant à la pause d'hésitation, on observe qu'elle 
joue un rôle pragmatique lorsqu'elle permet au locuteur de préparer ses idées. En ce qui concerne la pause de focalisation, on peut dire qu'elle est utilisée intentionnellement par l'homme politique kurde pour des raisons pragmatiques. Dans tous les exemples donnés, nous avons pu constater qu'il utilise les pauses de focalisation pour attirer l'attention des auditeurs sur un mot qui lui paraît important. Il en fait usage soit avant, soit après le mot focalisé. Nous avons dénombré 122 pauses de focalisation dans le discours politique de Nechirvan Barzani. Ce nombre nous parait important pour un discours de 23 minutes. Cet usage de la pause de focalisation est devenu une réelle stratégie discursive mise en place par les hommes politiques. Une stratégie qui attribue une efficacité accrue au discours politique.

\section{Bibliographie}

- BARZANI, Nechirvan (2011) Conférence de presse sur les événements de Saulaymanie le 16/02/2011[en ligne].Disponible sur http://www.youtube.com/watch?v=rzUOxhU8Df4(consulté le 25 février 2012).

- DISTER, Anne (2008), La notion subjective de la pause constitue-telle un bon indice pour le découpage de textes oraux ? M. Constant et al. (Éd.), Description Linguistique pour le Traitement Automatique du Français, Louvain: Presses Universitaires de Louvain, pp.165-186.

- DUEZ, Danielle (1999), La fonction symbolique des pauses dans la parole de l'homme politique, Faits de Langues, vol. $7, \mathbf{n}^{\circ} 13$, pp.91-97.

- $\quad$ LACHERET-DUJOUR, Anne \& BEAUGENDRE, Fréderic (1999), La Prosodie du Français, Paris : CNRS.

- MOREL, Mary- Annick \& DANON-BOILEAU, Laurent (1998), Grammaire de l'Intonation: l'exemple du français oral, Paris : Ophrys.

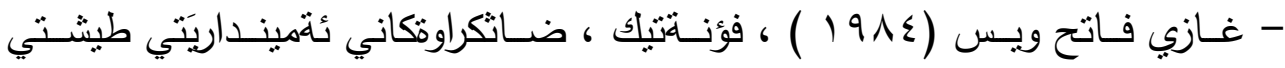

$$
\begin{aligned}
& \text { رؤشتنبيري و لاوان، زنجيرةي ذمارة ه ، بةغدا. }
\end{aligned}
$$

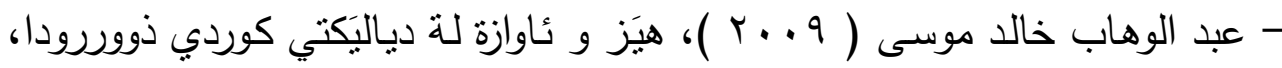

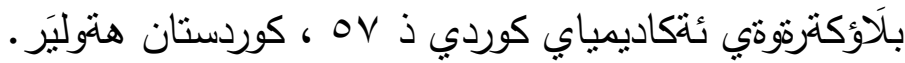

